\title{
Excited state systematics in extracting nucleon electromagnetic form factors from the lattice
}

\author{
S. Capitani ${ }^{1,2}$, M. Della Morte ${ }^{3}$, G. von Hippel ${ }^{1}$, B. Jäger ${ }^{1,2}$, B. Knippschild ${ }^{1}$, \\ H.B. Meyer ${ }^{1,2}$,T.D. Rae ${ }^{* \dagger 1}$, H. Wittig ${ }^{1,2}$ \\ ${ }^{1}$ PRISMA Cluster of Excellence and Institut für Kernphysik, Becher-Weg 45, University of \\ Mainz, D-55099 Mainz, Germany \\ ${ }^{2}$ Helmholtz Institute Mainz, University of Mainz, D-55099 Mainz, Germany \\ ${ }^{3}$ IFIC, c/ Catedrático José Beltrán, 2, 46980 Valencia, Spain \\ E-mail: thraeduni-mainz.de
}

Nucleon form factors are central observables of hadronic physics and provide details of the nucleon's distribution of charge and magnetisation. Currently, lattice simulations fall short of the accuracy achieved by experiment. Furthermore, the simulations of the nucleon electromagnetic form factor fail to reproduce experimental results [1, 2]; thus it is important to ensure that systematic effects are under control in lattice simulations.

We present recent results for the nucleon electromagnetic (EM) form factors using lattice QCD, including the determination of the charge radii. The standard approach is to extract the form factors via a plateau fit to the lattice data using a 'large-enough' time separation between the operators at the source and sink. To check that this removes excited state contaminations to an acceptable level, we employ two further extraction methods: a fit that explicitly accounts for the contamination; and the use of a summed operator insertion, which suppresses the contamination. A comparison of the methods allows for the study of systematic effects related to excited state contributions entering in the $Q^{2}$ dependence of the form factors. This work (also presented in [3]) provides an update on results previously presented in [4] and follows the methodology used in a recent study of the nucleon's axial form factor [5]. Similar methods have been used in [6]. Our simulations use non-perturbatively $\mathscr{O}(a)$ improved Wilson fermions in $N_{f}=2$ QCD, measured on the CLS ensembles. Further details of the lattice ensembles used may also be found in [3].

Xth Quark Confinement and the Hadron Spectrum

8-12 October 2012

TUM Campus Garching, Munich, Germany

\footnotetext{
*Speaker.

${ }^{\dagger}$ Supported by DFG grant HA4470/3-1
} 
The matrix element of a nucleon interacting with an EM current $V^{\mu}$ may be decomposed as:

$$
\left\langle N\left(p^{\prime}, s^{\prime}\right)\left|V_{\mu}\right| N(p, s)\right\rangle=\bar{u}\left(p^{\prime}, s^{\prime}\right)\left[\gamma_{\mu} F_{1}\left(Q^{2}\right)+i \frac{\sigma_{\mu v} q_{v}}{2 m_{N}} F_{2}\left(Q^{2}\right)\right] u(p, s)
$$

where $F_{1}$ and $F_{2}$ are the Dirac and Pauli form factors. These form factors are related to the Sachs form factors, $G_{E}$ and $G_{M}$, that are measured in scattering experiments via the differential cross section. The lattice calculation ${ }^{1}$ of the form factors requires a ratio of correlation functions,

$$
R_{\gamma_{\mu}}\left(\vec{q}, t, t_{s}\right)=\frac{C_{3, \gamma_{\mu}}\left(\vec{q}, t, t_{s}\right)}{C_{2}\left(\overrightarrow{0}, t_{s}\right)} \sqrt{\frac{C_{2}\left(\vec{q}, t_{s}-t\right) C_{2}(\overrightarrow{0}, t) C_{2}\left(\overrightarrow{0}, t_{s}\right)}{C_{2}\left(\overrightarrow{0}, t_{s}-t\right) C_{2}(\vec{q}, t) C_{2}\left(\vec{q}, t_{s}\right)}}
$$

$C_{2}(\vec{p}, t)$ and $C_{3, \gamma_{\mu}}\left(\vec{q}, t, t_{s}\right)$ are two- and three-point functions respectively containing suitably chosen interpolating operators with the correct quantum numbers to create a nucleon and, in the case of the three-point function, also the conserved/local vector current. $G_{E}$ and $G_{M}$ may be extracted for large $t$ :

$$
R_{\gamma_{0}}(\vec{q})=G_{E}\left(Q^{2}\right) \sqrt{(M+E) / 2 E}, \quad R_{\gamma_{i}}(\vec{q})=\varepsilon_{i j} p_{j} G_{M}\left(Q^{2}\right) \sqrt{1 /(2 E(E+M))} .
$$

Correlation functions must have reached their asymptotic behaviour for a reliable and unbiased determination of the form factors. However, we observe exponentially decaying excited states from both the source and sink. Therefore, simple plateau fits show a trend towards higher values for small source-sink separations, i.e. for decreasing $t_{s}$. To control systematics, it is important to take these excited states into account. Contributions to the ratio from the ground and excited states may be factorised, $R\left(\vec{q}, t, t_{s}\right)=R^{0}\left(\vec{q}, t, t_{s}\right)\left(1+\mathscr{O}\left(e^{-\Delta t}\right)+\mathscr{O}\left(e^{-\Delta^{\prime}\left(t_{s}-t\right)}\right)\right)$, where $\Delta$ and $\Delta^{\prime}$ are the excitation energies of the initial and final nucleons respectively. We can take the excited states into account using a fit to $R\left(\vec{q}, t, t_{s}\right)=G_{E, M}+b_{1} e^{-\Delta t}+b_{2} e^{-\Delta\left(t_{s}-t\right)}+b_{3} e^{-\Delta t_{s}}$, where we assume $\Delta=\Delta^{\prime}$ and we fix $\Delta=2 m_{\pi}$. An alternative that is not based upon this assumption, uses summed operator insertions [7]:

$$
S\left(t_{s}\right)=\sum_{t=0}^{t_{s}} R\left(\vec{q}, t, t_{s}\right) \rightarrow c\left(\Delta, \Delta^{\prime}\right)+t_{s}\left(G_{E, M}+\mathscr{O}\left(e^{-\Delta t_{s}}\right)+\mathscr{O}\left(e^{-\Delta^{\prime} t_{s}}\right)\right) .
$$

This allows the form factors to be extracted from the slope, by computing $S\left(t_{s}\right)$ for several $t_{s}$, resulting in more suppressed excited states because $t_{s}>t,\left(t_{s}-t\right)$.

In order to model the $Q^{2}$ dependence of the form factors, a dipole ansatz is adopted, from which the charge radius is extracted. We may obtain the magnetic moment $\mu$ from $G_{M}(0)$ or

$$
G_{E, M}\left(Q^{2}\right)=G_{E, M}(0) /\left(1+Q^{2} / M_{E, M}^{2}\right)^{2}, \quad \mu=\lim _{Q^{2} \rightarrow 0} \frac{G_{M}\left(Q^{2}\right)}{G_{E}\left(Q^{2}\right)} .
$$

Thus far, we have sketched the methodology commonly employed to extract form factors on the lattice. Using this, we study the systematics of the extraction by separately employing the three analyses described above: a plateau fit (for the largest $t_{s}$ ), a simultaneous excited state fit to $t$ and $t_{s}$ and the summation method. A comparison of the three methods in fig. 1 (left panel) shows that

\footnotetext{
${ }^{1}$ We concentrate on the conserved vector current (as this removes the need for renormalisation of the lattice operators) and consider the iso-vector case, for which quark-disconnected diagrams cancel. To improve the overlap of the interpolating operators with the nucleon, we use Gaussian smearing at both the source and sink and HYP smeared links.
} 

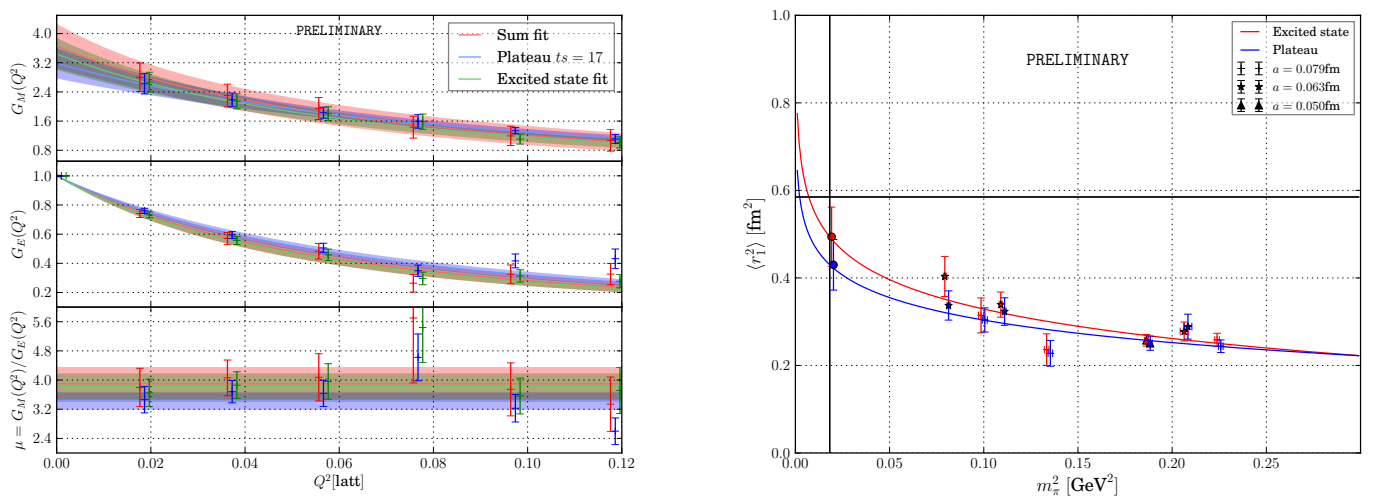

Figure 1: Left panel: Results for our most chiral $\left(m_{\pi}=277 \mathrm{MeV}\right)$ lattice. The top two panels show the $Q^{2}$ dependence for $G_{E}$ and $G_{M}$. The bottom panel shows a determination of the magnetic moment, $\mu$. This is shown for the three extraction methods. Right panel: Chiral extrapolation of the Dirac radius to the physical point (vertical black line) using $\left\langle r_{1}^{2}\right\rangle=c_{1}+c_{2} \log \left(m_{\pi}^{2}\right)$ using the largest $t_{s}$ plateau-fit (blue) and excited state fit (red) for the extraction. The horizontal black line shows the experimental result. The different symbols indicate the lattice spacing (see legend) and the leftmost two points show our extrapolated values.

whilst all three methods agree within statistical errors, we observe a systematic trend for better agreement between the two methods that account for excited state effects. This is echoed in all of our ensembles $\left(m_{\pi}=277\right.$ to $\left.649 \mathrm{MeV}\right)$. In order to perform an extrapolation in the pion mass to the physical point, we model the chiral behaviour of the radii using a $\mathrm{HB} \chi \mathrm{PT}$ inspired fit [8]. Fig. 1 (right panel) compares the determination of the Dirac radius $\left\langle r_{1}^{2}\right\rangle$ from an excited state fit with the plateau fit. We observe an increase in the central value for all but one ensemble and also that the difference increases as the pion mass decreases (excited states are expected to contribute more for more chiral $m_{\pi}$ ), resulting in a larger radius closer to the experimental result. We have also looked at $\kappa=\mu-1$ and $\left\langle r_{2}^{2}\right\rangle$, for which we see a similar behaviour. These are however all at the preliminary stage. The large statistical errors for all methods highlight the need for greater statistics and for more chiral points to be added. Monte Carlo ensembles exist for more chiral points (the lightest $\sim 200 \mathrm{MeV}$ ), but are yet to be analysed. The results indicate the importance of excited states and that they should be a consideration in studies of potential systematic effects.

\section{References}

[1] C. Alexandrou, PoS LATTICE 2010 (2010) 001 [arXiv:1011.3660 [hep-lat]].

[2] D. B. Renner, PoS LAT 2009 (2009) 018 [arXiv:1002.0925 [hep-lat]].

[3] S. Capitani, M. Della Morte, et al., PoS LATTICE 2012 (2012) 177 arXiv:1211.1282 [hep-lat].

[4] S. Capitani, B. Knippschild, et al., PoS LATTICE 2010 (2010) 147 [arXiv:1011.1358 [hep-lat]].

[5] S. Capitani, M. Della Morte, et al., Phys. Rev. D 86 (2012) 074502 [arXiv:1205.0180 [hep-lat]].

[6] J. R. Green, M. Engelhardt, et al., arXiv:1209.1687 [hep-lat].

[7] L. Maiani, G. Martinelli, et al., Nucl. Phys. B 293, 420 (1987).

[8] A. A. Khan, M. Göckeler, et al., Phys. Rev. D 74 (2006) 094508 [hep-lat/0603028]. 